\title{
Risk-stratified surveillance and cost effectiveness of follow-up after radical cystectomy in patients with muscle-invasive bladder cancer
}

\author{
Ayumu Kusaka ${ }^{1}$, Shingo Hatakeyama ${ }^{1}$, Shogo Hosogoe ${ }^{1}$, Itsuto Hamano ${ }^{1}$, Hiromichi \\ Iwamura $^{1}$, Naoki Fujita ${ }^{1}$, Ken Fukushi' ${ }^{1}$, Takuma Narita ${ }^{1}$, Kazuhisa Hagiwara ${ }^{1}$, \\ Hayato Yamamoto ${ }^{1}$, Yuki Tobisawa ${ }^{1}$, Tohru Yoneyama ${ }^{2}$, Takahiro Yoneyama ${ }^{1}$, \\ Yasuhiro Hashimoto ${ }^{2}$, Takuya Koie ${ }^{1}$, Hiroyuki Ito ${ }^{3}$, Kazuaki Yoshikawa ${ }^{4}$, Toshiaki \\ Kawaguchi ${ }^{5}$ and Chikara Ohyama ${ }^{1,2}$ \\ ${ }^{1}$ Department of Urology, Hirosaki University Graduate School of Medicine, Hirosaki, Japan \\ ${ }^{2}$ Department of Advanced Transplant and Regenerative Medicine, Hirosaki University Graduate School of Medicine, Hirosaki, \\ Japan \\ ${ }^{3}$ Department of Urology, Aomori Rosai Hospital, Hachinohe, Japan \\ ${ }^{4}$ Department of Urology, Mutsu General Hospital, Mutsu, Japan \\ ${ }^{5}$ Department of Urology, Aomori Prefectural Central Hospital, Aomori, Japan
}

Correspondence to: Shingo Hatakeyama, email: shingoh@hirosaki-u.ac.jp

Keywords: medical cost, radical cystectomy, recurrence, screening, surveillance

Received: March 07, 2017 Accepted: June 20, 2017

Published: July 06, 2017

Copyright: Kusaka et al. This is an open-access article distributed under the terms of the Creative Commons Attribution License 3.0 (CC BY 3.0), which permits unrestricted use, distribution, and reproduction in any medium, provided the original author and source are credited.

\section{ABSTRACT}

Background: The recurrence risk stratification and the cost effectiveness of oncological surveillance after radical cystectomy are not clear. We aimed to develop a risk stratification and a surveillance protocol with improved cost effectiveness after radical cystectomy.

Results: Of 581 enrolled patients, 175 experienced disease recurrences. The pathology-based protocol presented significant differences in recurrence-free survival between normal- and high-risk patients, but the medical expense was high, especially in normal-risk ( $\leq$ pT2pNO) patients. Cox regression analysis identified six factors associated with recurrence-free survival. Risk score-based 5-year follow-up was significantly more cost effective than the pathology-based protocol.

Materials and Methods: We retrospectively evaluated 581 patients with radical cystectomy for muscle-invasive bladder cancer at $\mathbf{4}$ hospitals. Patients with routine oncological follow-up were stratified into normal- and high-risk groups by a pathologybased protocol utilizing pT, pN, lymphovascular invasion, and histology. Cost effectiveness of the pathology-based protocol was evaluated and a risk-score-based protocol was developed to optimize cost effectiveness. Risk-scores were calculated by summing risk factors independently associated with recurrence-free survival. Patients were stratified by low-, intermediate-, and high-risk score. Estimated cost per one recurrence detection by the pathology and by risk-scores were compared.

Conclusions: Risk-score-stratified surveillance protocol has potential to reduce over-evaluation after radical cystectomy without adverse effects on medical cost. 


\section{INTRODUCTION}

Radical cystectomy (RC) with extended pelvic lymph node dissection is the standard treatment for non-metastatic muscle-invasive bladder cancer (MIBC) $[1,2]$. Long-term outcome and predictors of disease relapse after RC are well documented [1-4]. Despite advances in treatment, an estimated $38 \%-49 \%$ of patients experience recurrence within 10 years even at high-volume institutes $[1,2,4]$. The prognosis is dismal following recurrence. Various surveillance regimens have been proposed [4-7], but the most effective followup frequency and methods of evaluation are unclear. In addition, evidence is lacking on the cost effectiveness of routine oncological follow-up to detect recurrence after $\mathrm{RC}[4,8]$ and needs investigation. The main purposes of the present study are to develop the risk stratification and the optimal surveillance protocol for tumor recurrence that improves cost effectiveness after RC. First, we estimated the per-capita cost of detecting post-RC recurrence of MIBC using an existing pathology-based protocol (Table 1 , upper rows). We then developed a novel risk-scorebased protocol using Cox proportional hazard regression and compared the cost effectiveness of the pathologybased and risk-score-based protocols.

\section{RESULTS}

Of the 581 patients with RC, 299 (52\%) were included in the pathology-based normal-risk group and $282(48 \%)$ were included in the high-risk group. The clinicopathological characteristics are shown in Table 2. Patient sex ratio, preoperative eGFR, $\geq \mathrm{cT} 3$, surgical margin positive $(\mathrm{SM}+), \mathrm{cN}+$, neoadjuvant chemotherapy (NAC), type of urinary diversion, and tumor recurrence in the normal- and high-risk groups were significantly different. Because of a higher rate of relapse, median follow-up was significantly shorter in the high-risk (33 months) than in the normal-risk (60 months) group. Recurrence-free survival in the 2 groups was also significantly different (Figure 1A). The number of patients with symptomatic tumor recurrence was 100/175 (57\%). There was no significant difference in the number of patients with symptomatic recurrence between the normalrisk 28/43 (65\%) and high-risk 72/132 (55\%) groups $(P=0.291)$. The number of patients with tumor recurrence within 24 months in the high-risk group ( $n$ $=109 / 133,82 \%)$ and the normal-risk group $(\mathrm{n}=30 / 42$, $71 \%)$ were not significantly different $(P=0.189$, Figure $1 \mathrm{~B})$. The estimated cost per one recurrence detection was extremely high at 3,54 , and 60 months $(>\$ 100,000)$ in the normal-risk group (Figure 1C). The 12-month cost effectiveness was more acceptable in the high-risk group $(<\$ 10,000)$, but gradually increased thereafter (Figure 1C). The median estimated cost per one recurrence detection was significantly higher in the normal-risk
$(\$ 65,736)$ group than in the high-risk $(\$ 18,775)$ group $(P=0.0051)$ (Figure 2A)

Multivariate Cox regression analysis found 6 factors that were independently associated with recurrence-free survival, including $\mathrm{LVI}+, \mathrm{pN}+, \geq \mathrm{pT} 3$ or $\mathrm{SM}+$, urinary diversion (non-neobladder), preoperative chronic kidney disease (CKD), and cardiovascular disease (CVD) (Table 3, Figure 3A). The risk-scores were calculated by adding each independent risk factor for recurrence-free survival (risk score ranged 0-6). Patients were divided by their score into low-risk $(0-1)$, intermediate-risk (2-3), and high-risk (4-6) groups (Table 4). Recurrence-free survival was significantly shorter in patients with highrisk than with low-risk $(P<0.001)$ or intermediaterisk $(P<0.001)$ scores (Figure 1D). The number of patients who experienced tumor recurrence within 24 months was significantly higher in high-risk-score $(\mathrm{n}=$ $65 / 71,92 \%)$ than in the low-risk score ( $\mathrm{n}=5 / 33,15 \%$, $P<0.001)$ or intermediate-risk score $(\mathrm{n}=40 / 71,56 \%$, $P<0.001$ ) groups (Figure 1E). The number of patients with symptomatic tumor recurrence in the low-risk-score, intermediate-risk score, and high-risk score were 18/33 (55\%), 41/71 (58\%), and 41/71 (58\%), respectively. Based on this risk-stratification, we developed a risk score-based protocol for oncological follow-up (Table 1, lower rows), and evaluated per-capita cost of recurrence detection (Figure 1F). The median estimated cost per one recurrence detection was significantly different between the low-risk $(\$ 39,960)$ and intermediate-risk $(\$ 16,766)$ groups $(P=$ $0.0042)$, the low-risk and high-risk $(\$ 10,209)$ groups $(P=0.0011)$, and the intermediate-risk and high-risk, groups $(P=0.0256)$ (Figure 2B). The risk-score-based protocol led to a dramatic cost reduction compared to the pathology-based protocol. The total estimated 5-year screening cost was 1.9-fold higher with the pathologybased protocol $(\$ 1,148,687)$ than with the risk score-based protocol $(\$ 613,901)$. The estimated cost difference was $\$ 534,786$ for 5 years (Figure $2 \mathrm{C}$ ). The number of patients that potentially fails in detection using the risk-scorebased protocol was $4(12 \%)$ in the low, $3(4.2 \%)$ in the intermediate, and $1(1.4 \%)$ in the high-risk-score group (Figure 3B).

\section{DISCUSSION}

Currently, there is no strong evidence or consensus on how to appropriately follow-up RC patients after surgical intervention [9], and a few studies have been conducted to investigate the cost effectiveness of surveillance regimens after RC [8]. The effectiveness of a stage-based approach to tumor surveillance after $\mathrm{RC}$ has been shown $[10,11]$. Yafi et al. reported the effectiveness of a stage-based protocol $(\leq \mathrm{pT} 2 \mathrm{pN} 0$, pT3-4pN0, pTxpN+) that captured most recurrences while limiting over investigation using pooled data from 2287 patients in 8 Canadian academic centers [4]. 
Table 1: Pathology-based and risk-score-based protocol for oncological follow-up

\begin{tabular}{|c|c|c|c|c|c|c|c|c|c|c|c|c|c|c|}
\hline Pathology-based protocol & & & & & & & Ion & afte & $\mathbf{R C}$ & & & & & \\
\hline Normal-risk & 3 & 6 & 9 & 12 & 15 & 18 & 21 & 24 & 30 & 36 & 42 & 48 & 54 & 60 \\
\hline Ultrasonography & $\bullet$ & $\bullet$ & $\bullet$ & $\bullet$ & o & $\bullet$ & & $\bullet$ & $\bullet$ & $\bullet$ & $\bullet$ & $\bullet$ & $\bullet$ & $\bullet$ \\
\hline Urine cytology & $\bullet$ & $\bullet$ & $\bullet$ & $\bullet$ & ० & $\bullet$ & & $\bullet$ & & $\bullet$ & & $\bullet$ & & $\bullet$ \\
\hline Blood and serum test & $\bullet$ & $\bullet$ & & $\bullet$ & & $\bullet$ & & $\bullet$ & & $\bullet$ & & $\bullet$ & & $\bullet$ \\
\hline CT scan & & $\bullet$ & & $\bullet$ & & $\bullet$ & & $\bullet$ & & $\bullet$ & & $\bullet$ & & $\bullet$ \\
\hline High-risk & 3 & 6 & 9 & 12 & 15 & 18 & 21 & 24 & 30 & 36 & 42 & 48 & 54 & 60 \\
\hline Ultrasonography & $\bullet$ & $\bullet$ & $\bullet$ & $\bullet$ & $\bullet$ & $\bullet$ & $\bullet$ & $\bullet$ & & $\bullet$ & & $\bullet$ & & $\bullet$ \\
\hline Urine cytology & $\bullet$ & $\bullet$ & $\bullet$ & $\bullet$ & $\bullet$ & $\bullet$ & $\bullet$ & $\bullet$ & & $\bullet$ & & $\bullet$ & & $\bullet$ \\
\hline Blood and serum test & $\bullet$ & $\bullet$ & $\bullet$ & $\bullet$ & $\bullet$ & $\bullet$ & $\bullet$ & $\bullet$ & & $\bullet$ & & $\bullet$ & & $\bullet$ \\
\hline CT scan & $\bullet$ & $\bullet$ & $\bullet$ & $\bullet$ & $\bullet$ & $\bullet$ & $\bullet$ & $\bullet$ & $\bullet$ & $\bullet$ & $\bullet$ & $\bullet$ & $\bullet$ & $\bullet$ \\
\hline Risk-score-based protocol & \multicolumn{14}{|c|}{ Months after RC } \\
\hline Low-risk & 3 & 6 & 9 & 12 & 15 & 18 & 21 & 24 & 30 & 36 & 42 & 48 & 54 & 60 \\
\hline Ultrasonography & & $\bullet$ & & $\bullet$ & & & & $\bullet$ & & $\bullet$ & & $\bullet$ & & $\bullet$ \\
\hline Urine cytology & & $\bullet$ & & - & & & & $\bullet$ & & $\bullet$ & & $\bullet$ & & $\bullet$ \\
\hline Blood and serum test & & $\bullet$ & & $\bullet$ & & & & $\bullet$ & & $\bullet$ & & $\bullet$ & & $\bullet$ \\
\hline CT scan & & & & $\bullet$ & & & & & & $\bullet$ & & & & \\
\hline Intermediate- and high-risk & 3 & 6 & 9 & 12 & 15 & 18 & 21 & 24 & 30 & 36 & 42 & 48 & 54 & 60 \\
\hline Ultrasonography & $\bullet$ & $\bullet$ & $\bullet$ & $\bullet$ & & $\bullet$ & & $\bullet$ & & $\bullet$ & & $\bullet$ & & $\bullet$ \\
\hline Urine cytology & $\bullet$ & $\bullet$ & $\bullet$ & $\bullet$ & & $\bullet$ & & $\bullet$ & & $\bullet$ & & $\bullet$ & & $\bullet$ \\
\hline Blood and serum test & $\bullet$ & $\bullet$ & $\bullet$ & $\bullet$ & & $\bullet$ & & $\bullet$ & & $\bullet$ & & $\bullet$ & & $\bullet$ \\
\hline CT scan & $\bullet$ & $\bullet$ & $\bullet$ & $\bullet$ & $\bullet$ & $\bullet$ & $\bullet$ & $\bullet$ & & $\bullet$ & & $\bullet *$ & & $\bullet$ \\
\hline
\end{tabular}

*,only for the high-risk group, CT scan included chest, abdomen and pelvis. o, optional examination when indicated.

Although pathological stage is known to be a strong predictor of relapse, variations of recurrence pattern in those patients prevented effective screening with a universal surveillance protocol for all patients. Our results showed that a pathology-based screening protocol has potential to increase unnecessary testing in normal-risk $(\leq \mathrm{pT} 2 \mathrm{pN} 0)$ patients. Not only pathological outcome but other clinical risk factors have significant impact on tumor recurrence after RC. To address this, we first estimated the cost per one recurrence detection using a pathology-based protocol, which was found not optimal as a way to stratify patient recurrence risk for cost effective surveillance. We developed risk-score-based surveillance protocol and stratified the study patients into low-risk (0-1), intermediate-risk (2-3) and high-risk (4-6) score groups. The recurrence-free survival in each risk-score is shown in Figure 3C. Not only pathological outcomes, but also preoperative variables including CVD and CKD had a significant impact on recurrence after RC. Recent studies suggested the impact of CKD on prognosis in MIBC [12] and upper urinary tract urothelial cancer [13]. Patients with renal impairment are at risk of CVD [14], but the reason for the strong association between CKD and poor recurrence-free survival is not clear. Previous studies suggested kidney and urinary tract cancers are high risk for renal dysfunction due to the tumor location (obstruction, and/or reduction of nephron mass) $[13,15-17]$. The prevalence of CKD in genitourinary cancers were reported $8.7-21.4 \%[15,18]$. In the present study, the prevalence of CKD in MIBC patients was $37 \%$, which is a strong bias in our cohort. We investigated the relationship between CKD, CVD and prognosis. Our results suggested patients with CKD had significantly higher risk for recurrence and cancer death than the patients without CKD. Similarly, patients with CVD had significantly higher risk for recurrence and cancer 
death than the patients without CVD (Figure 5A, 5B). In addition, the patients with CKD had significantly higher risk-score ( $2.5 \pm 1.3$ points) than those without CKD (1.2 \pm 1.3 points, $P<0.001$ ) (Figure $5 \mathrm{C}$, left). Similarly, the patients with CVD had significantly higher risk-score (3.1 \pm 1.3 points $)$ than those without CVD $(1.6 \pm 1.4$ points, $P<0.001$ ) (Figure 5C, right). However, when simply compared cause of death, no difference was observed in the cause of death between patients with and without CKD (Figure 5D). In addition, there was no significant difference between the number of CKD patients with CVD $(n=35 / 68,52 \%)$ and without CVD $(n=218 / 513$, $42 \%$ ) (Figure $5 \mathrm{E}$ ). Therefore, as previous study suggested [18], bladder cancer is great potential to have a renal dysfunction during the disease progression. Although there is no clear explanation for that, the effects of metabolic syndrome [19] and chronic inflammation may explain the association of CKD and oncological outcome $[20,21]$. Long-term inflammation and oxidative stress caused by CKD and linked to organ degradation may increase carcinogenicity. The other potential factor that influencing on poor prognosis was frailty. Patients with CKD and CVD are more likely to be frail [22] because these are likely to exist in combination with comorbid conditions, disability, and polypharmacy [23, 24]. In addition, frailty is also one of the important parameters of cancers $[25,26]$. Recent study reported the association between frailty and inflammatory markers in elderly cancer patients [27]. Our previous study suggested renal function has potential to predict postoperative frailty [28]. Although we could not address the direct reason why CKD and CVD were independently influence on recurrence after $\mathrm{RC}$, these results suggest a potential relationship between cancer progression, CKD, CVD and frailty. However, evidence to confirm this hypothesis is lacking. Future studies to assess mechanisms underlying carcinogenesis and risk factors are warranted.

Age is also a well-known risk factor for recurrence. To investigate the relationship between age and recurrence pattern, we divided patients into two groups between younger $(\leq 70)$ and older $(>70)$. Our results suggested that there was no significant difference in the recurrence pattern between the younger $(\leq 70)$ and older $(>70)$ patients. However, late recurrence ( $>24$ months) showed marginal difference between $\leq 70$ years $(15 / 202,7 \%)$ and $>70$ years $(19 / 138,14 \%)(P=0.056)($ Figure $5 \mathrm{~F})$.

Inclusion of $\mathrm{cN}+$ for risk-stratification needs to be debated. Because standard lymph node dissection is recommended for all patients at $\mathrm{RC}$, we selected $\mathrm{pN}+$ for one of the risk factors. Although $\mathrm{cN}+$ is one of the risk
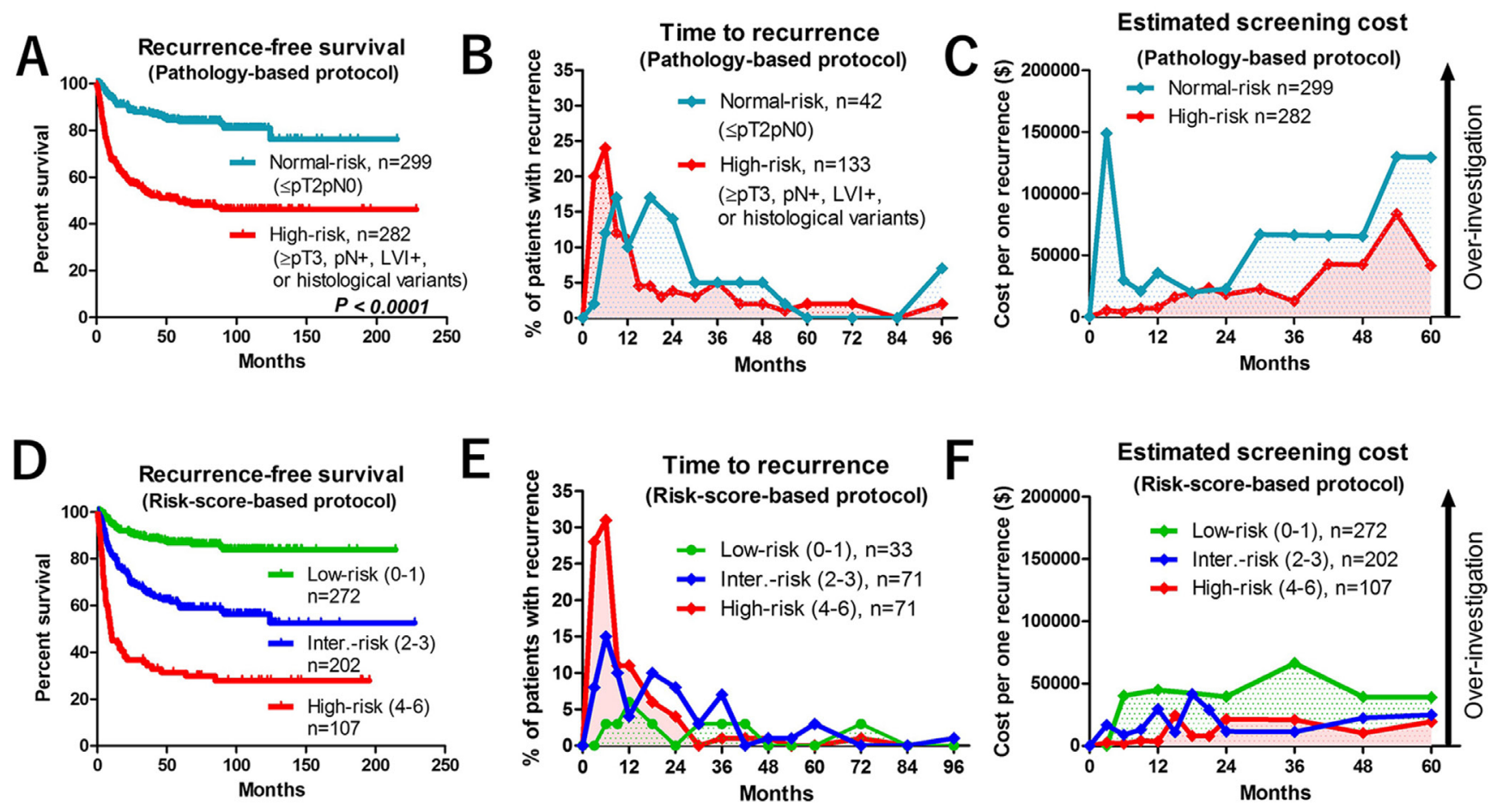

Figure 1: Oncological and economic outcomes of pathology-based and risk score-based surveillance protocols. (A) Recurrence-free survival in normal-risk $(\leq \mathrm{pT} 2 \mathrm{pN} 0)$ and high-risk $(\geq \mathrm{pT} 3, \mathrm{pN}+$, lymphovascular invasion [LVI +$]$, or histological variants) patients in the pathology-based protocol. (B) Time-course analysis of recurrence pattern of normal-risk and high-risk patients in the pathology-based protocol. (C) Estimated cost per one recurrence detection in normal-risk and high-risk patients in the pathology-based protocol. (D) Recurrence-free survival of low-risk score (score 0-1), intermediate-risk score (2-3), and high-risk score (4-6) patients. (E) Time-course analyses of recurrence in low-risk, intermediate-risk, and high-risk score patients. (F) Estimated cost per one recurrence detection in low-risk, intermediate-risk, and high-risk score patients. 


\begin{tabular}{|c|c|c|c|c|}
\hline & All & Normal-risk & High-risk & $P$ value \\
\hline$n$ & 581 & 299 & 282 & \\
\hline Age, years (IQR) & $69(62-75)$ & $69(62-74)$ & $69(62-75)$ & 0.694 \\
\hline Sex (Male), $n=$ & $458(79 \%)$ & $247(83 \%)$ & $221(75 \%)$ & 0.022 \\
\hline ECOG PS $>0, n=$ & $15(2.6 \%)$ & $5(1.7 \%)$ & $6(2.1 \%)$ & 0.767 \\
\hline Hypertension (HTN), $n=$ & $179(31 \%)$ & $92(31 \%)$ & $85(30 \%)$ & 0.870 \\
\hline Diabetes Mellitus (DM), $n=$ & $78(13 \%)$ & $47(16 \%)$ & $30(11 \%)$ & 0.425 \\
\hline Cardiovascular disease (CVD), $n=$ & $68(12 \%)$ & $25(8.4 \%)$ & $29(10 \%)$ & 0.071 \\
\hline Preoperative CKD, $n=$ & $215(37 \%)$ & $122(41 \%)$ & $131(46 \%)$ & 0.170 \\
\hline Preoperative eGFR (mL/min/1.73m²) & $66 \pm 20$ & $68 \pm 20$ & $63 \pm 19$ & 0.010 \\
\hline$\geq \mathrm{cT} 3, n=$ & $274(47 \%)$ & $101(34 \%)$ & $173(61 \%)$ & $<0.001$ \\
\hline Surgical margin positive $(\mathrm{SM}+), n=$ & $26(4.5 \%)$ & $0(0 \%)$ & $26(9.2 \%)$ & $<0.001$ \\
\hline cN positive, $n=$ & $44(7.6 \%)$ & $12(4.0 \%)$ & $32(11 \%)$ & 0.001 \\
\hline Neoadjuvant chemotherapy (NAC), $n=$ & $315(60 \%)$ & $197(66 \%)$ & $151(54 \%)$ & 0.002 \\
\hline Urinary diversion (Neobladder), $n=$ & $315(54 \%)$ & $195(75 \%)$ & $123(44 \%)$ & $<0.001$ \\
\hline Postoperative complication (Grade 3 or higher) & $15(2.6 \%)$ & $8(1.4 \%)$ & $7(1.2 \%)$ & 1.000 \\
\hline Non-urothelial carcinoma components included, $n=$ & $37(6.4 \%)$ & $0(0 \%)$ & $37(13 \%)$ & \\
\hline$\geq \mathrm{pT} 3, n=$ & $183(31 \%)$ & $0(0 \%)$ & $181(64 \%)$ & \\
\hline $\mathrm{pN}$ positive, $n=$ & $70(12 \%)$ & $0(0 \%)$ & $79(28 \%)$ & \\
\hline Lympho-vascular invasion (LVI), $n=$ & $195(34 \%)$ & $0(0 \%)$ & $194(69 \%)$ & \\
\hline Tumor recurrence, $n=$ & $175(30 \%)$ & $43(14 \%)$ & $132(47 \%)$ & $<0.001$ \\
\hline Median follow-up, months (IQR) & $45(13-90)$ & $60(23-95)$ & $33(9.7-75)$ & $<0.001$ \\
\hline
\end{tabular}

CKD: chronic kidney disease, SM: surgical margin.
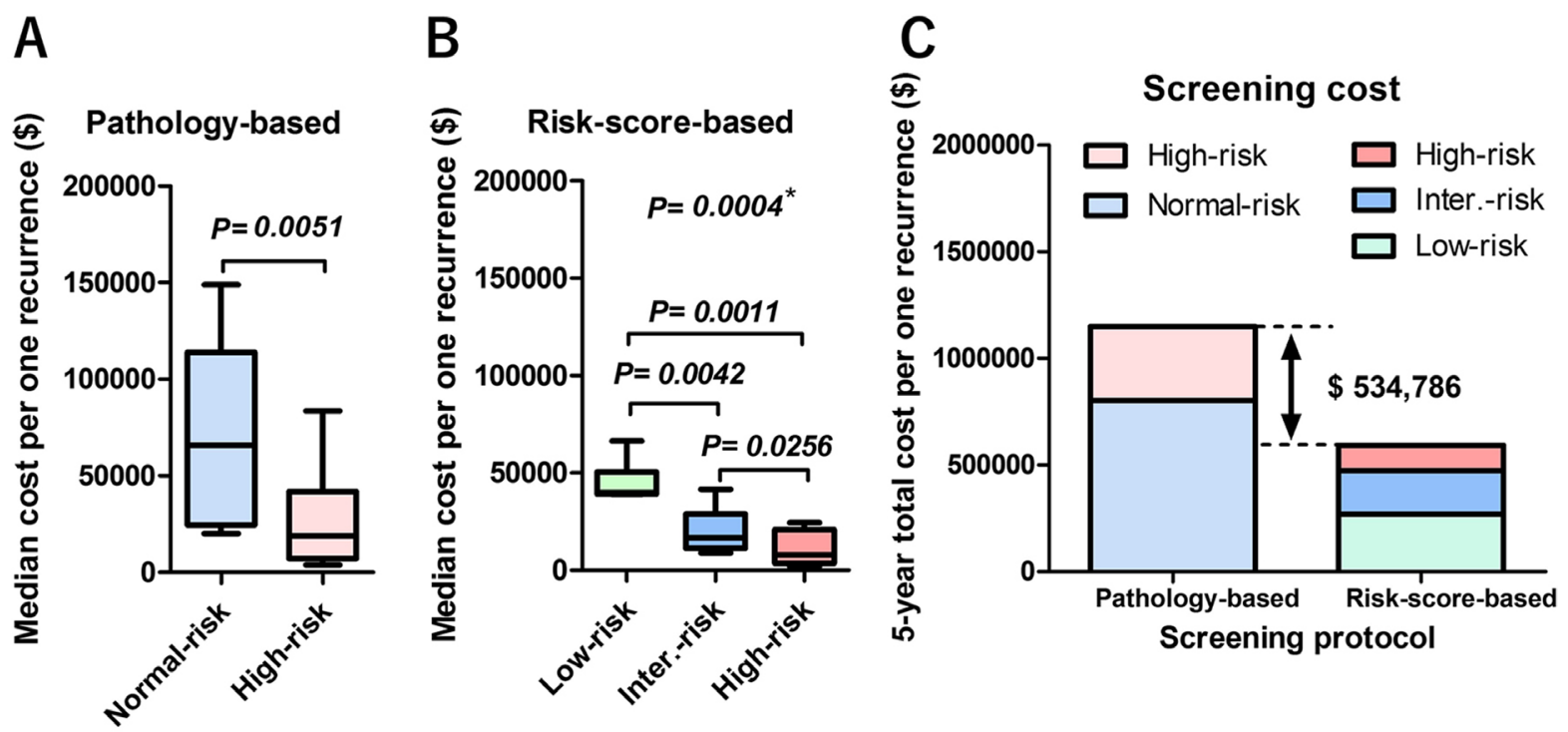

Figure 2: Per-capita cost to detect 5-year recurrence. (A) In the pathology-based protocol, median screening cost to detect one recurrence in 5 years was significantly higher in the normal-risk $(\$ 65,736)$ group than that of high-risk $(\$ 18,775)$ group $(P=0.0051)$. (B) In the risk score-based protocol, median screening cost to detect one recurrence in 5 years was significantly different in the three groups $(*$, Kruskal-Wallis test). There were significant differences between the low-risk $(\$ 39,960)$ and intermediate-risk $(\$ 16,766)$ groups $(P=0.0042)$, the low-risk and high-risk $(\$ 10,209)$ groups $(P=0.0011)$, and the intermediate-risk and high-risk groups $(P=0.0256) .(\mathbf{C})$ The estimated 5-year screening cost in the pathology-based protocol was $\$ 1,148,687$, whereas it was $\$ 613,901$ in the risk score-based protocol. The estimated difference was $\$ 534,786$ over 5 years. 
Table 3: Multivariate analysis for progression-free survival

\begin{tabular}{lcccc}
\hline & Risk & P value & HR & $\mathbf{9 5 \%}$ CI \\
\hline Age & Continuous & 0.550 & 1.01 & $0.99-1.02$ \\
Sex & Male & 0.703 & 0.93 & $0.65-1.34$ \\
ECOG PS & $>0$ & 0.995 & 1.00 & $0.41-2.40$ \\
Hypertension (HTN) & Positive & 0.090 & 1.34 & $0.96-1.89$ \\
Diabetes Mellitus (DM) & Positive & 0.969 & 1.01 & $0.63-1.63$ \\
Cardiovascular disease (CVD) & Positive & 0.042 & 1.55 & $1.02-2.36$ \\
Preoperative CKD & Positive & 0.001 & 1.66 & $1.22-2.26$ \\
Neoadjuvant chemotherapy (NAC) & Underwent & 0.526 & 1.11 & $0.81-1.51$ \\
Histology & Variants included & 0.142 & 1.50 & $0.87-2.59$ \\
Urinary diversion & Non-neobladder & 0.049 & 1.39 & $1.00-1.92$ \\
Pathological T stage & $\geq$ pT3 or SM+ & $<0.001$ & 1.99 & $1.40-2.84$ \\
Pathological N stage & pN positive & $<0.001$ & 2.15 & $1.47-3.16$ \\
Lymphovascular invasion & Positive & $<0.001$ & 2.32 & $1.61-3.35$ \\
\hline
\end{tabular}

CKD: chronic kidney disease, SM: surgical margin

factors for poor prognosis (Figure 4A, 4B, and Table 3), the clinical implication of $\mathrm{cN}+$ remains unclear because not all $\mathrm{cN}+$ predicts tumor relapse. In the present study, only $17 / 44(39 \%)$ patients with $\mathrm{cN}+$ had $\mathrm{pN}+$ by standard lymph node dissection. Of $27 / 44(61 \%)$ patients with $\mathrm{cN}+/ \mathrm{pN}-, 11$ patients $(25 \%)$ experienced recurrence. Three patients with $\mathrm{cN}+/ \mathrm{pN}-(6.8 \%)$ had lymph nodes recurrence alone while remain $8(18 \%)$ had systemic
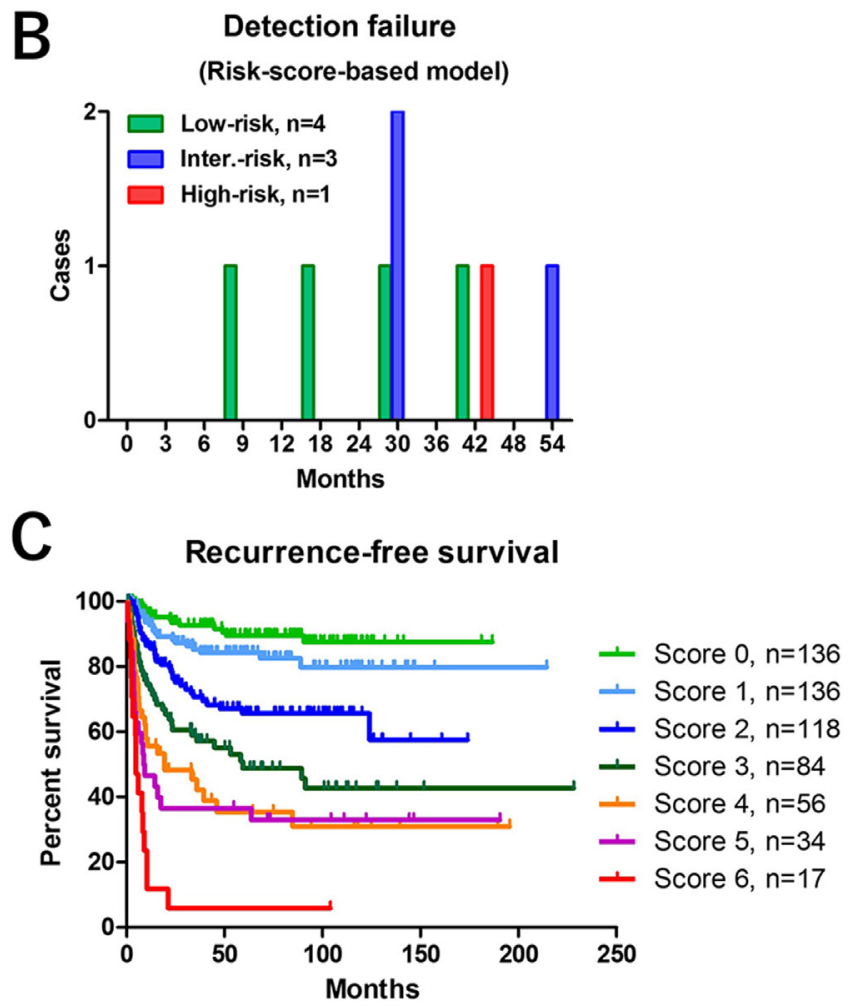

Figure 3: Risk factors selection and the impact of risk-scores on prognosis. (A) Hazard ratio and $95 \% \mathrm{CI}$ in multivariate Cox regression analysis. (B) The number of patients potentially undetected by the risk score based protocol were $4(12 \%)$ in the low-, $3(4.2 \%)$ in the intermediate- and $1(1.4 \%)$ in the high-risk group. (C) Recurrence-free survival was shown in each risk score. Patients were stratified into three groups by low-risk score (0-1), intermediate-risk score (2-3), and high-risk score (4-6), indicating the probability of relapse. LVI: lymphovascular invasion, SM: surgical margin, CKD: chronic kidney disease, CVD: cardiovascular disease, HTN: hypertension, NAC: neoadjuvant chemotherapy, DM: diabetes mellitus 
Table 4: Risk-score-based classification

\begin{tabular}{lcc}
\hline \multicolumn{1}{c}{ Variable } & Status & Risk score \\
\hline Cardiovascular disease & Positive & 1 \\
Preoperative CKD & Positive & 1 \\
Urinary diversion & Non-neobladder & 1 \\
Pathological T stage & $\geq \mathrm{pT}$ or SM+ & 1 \\
Pathological N stage & pN positive & 1 \\
Lymphovascular invasion & Positive & 1 \\
\hline Risk-score-based classification & & Sum of risk-score \\
\hline Low-risk & & $0-1$ \\
Intermediate-risk & & $2-3$ \\
High-risk & & $4-6$ \\
\hline
\end{tabular}

CKD: chronic kidney disease, SM: surgical margin.

recurrence (Figure 4C). On the other hand, 52/537 (9.7\%) patients with $\mathrm{cN}-$ had $\mathrm{pN}+$ by the standard lymph node dissection. In addition, the influence of NAC for $\mathrm{pN}$ status need to be addressed. We evaluated the difference of $\mathrm{pN}$ status between $\mathrm{cN}$ - patients treated with $\mathrm{NAC}$ and without $\mathrm{NAC}$, and $\mathrm{pN}$ status between $\mathrm{cN}+$ treated with NAC and without NAC. The result showed there were no significant difference of $\mathrm{pN}+$ between $\mathrm{cN}$ - patients treated with NAC (31/316, 9.8\%) and without NAC (21/221, 9.5\%) $(\mathrm{P}=0.914)$, and $\mathrm{pN}+$ between $\mathrm{cN}+$ treated with NAC $(12 / 32,38 \%)$ and without NAC $(5 / 12,42 \%)(P=$ 0.867 ) (Figure 4D). These results suggested the inaccuracy of $\mathrm{cN}+$ to predict pathological lymph node involvement, although we could not exclude false negative of standard lymph node dissection. Based on these limitations, we included $\mathrm{pN}+$ for the risk-stratification in the present study. However, further studies are needed to address the inclusion of $\mathrm{cN}+$ for risk-stratification model.

In the present study, type of urinary diversion was selected one of independent predictors for disease recurrence. This result suggested possibilities that the strong selection bias for urinary diversion and/or opportunity difference for surveillance after surgery. There is a strong selection bias for orthotopic bladder substitution including younger age, good performance status, and lower-risk for local recurrence. Due to the retrospective analysis, we could not assess the influence of frequent care in patients with stoma might have impact of follow-up surveillance. Further prospective studies are necessary to address these important clinical questions.

The use of neoadjuvant chemotherapy (NAC) for patients with renal impairment remains unclear [29-33]. Based on several guidelines [34, 35] NAC was not recommended in the patients with CKD (= cisplatin-unfit) and carboplatin-based regimen was believed less effective than cisplatin. However, no clear evidence currently supports the superiority of cisplatinbased regimens over carboplatin-based regimens in the neoadjuvant setting [36]. Therefore, we used short-term (two courses) carboplatin-based NAC for patients with $\mathrm{CKD}$, and performed RC within 90 days [37]. Indeed, our previous studies with short-term carboplatin-based NAC with immediate cystectomy suggested potential benefit of carboplatin based NAC for MIBC patients with preoperative CKD [30, 32, 33, 38]. Based on these results, although two NAC courses might be insufficient, we believe our protocol did not harm to prognosis in MIBC patients with CKD.

Although use of this risk score-based protocol may reduce over evaluation, the cost was still substantial because of the low relapse rate in the low-risk group. This result highlights the limitation of predicting relapse risk exclusively on clinical information. For a better understanding of tumor biology, biomarkers that predict malignant potential are necessary. Genome-based molecular classification is a potential biomarker $[39,40]$. A basal MIBC type has been associated with decreased disease-specific and overall survival because of its invasiveness and metastatic potential at presentation [39]. There is an urgent need to identify a molecular biomarker to inform clinical management.

This study was limited by its retrospective design. First, as we could not control all variables, it was subject to selection bias and the influence of confounding factors including all medical costs for oncological surveillance, $\mathrm{CT}$ cost for with or without a contrast medium, numbers of dissected and positive lymph nodes, smoking status, regimen of NAC and the influence of adjuvant/salvage therapy. In addition, we could not address the optimal timing and utility of urine cytology for routine screening in the present study because only five patients $(2.9 \%)$ were detected by urine cytology. Furthermore, we could not control the influence of frequent care in patients with stoma that might have impact of follow-up surveillance. Second, we focused two points; risk stratification and cost effectiveness of postoperative follow-up. In this point of view, it might be hard to discuss these two issues at the same time. However, when we simply evaluated 
the medical cost based on guideline recommended pathological risk factors, we found that pathologybased protocol did not improve the cost-effectiveness. In addition, it is not optimal for a clinical practice to use all previously reported risk factors. Therefore, we selected risk factors that significantly associated with tumor progression in our patients, and then developed the optimal risk stratification and surveillance protocol. Third, we could not clearly explain why CVD and CKD were selected as significant factors for recurrence in MIBC patients who underwent RC. Because there are not enough evidences to support the clinical implication of CVD and CKD for tumor progression, further basic and clinical research are necessary. Forth, the risk scorebased surveillance model might increase the number of patients not detected by screening. Finally, the implication of this study may not suitable for other population such as non-Asian people because the entire Japanese population is covered by universal health insurance (maximum copayment of $10 \%$ to $30 \%$ ). However, it is not easy to make the one-fit-all surveillance protocol due to medical system differences among the nations. Therefore, our study provides the idea that risk stratification in each nation is necessary to improve the cost-effectiveness in each medical system.

Despite these limitations, this study adds to our knowledge of the cost effectiveness of oncological surveillance after RC. Although the importance of riskstratified surveillance protocol has been reported, no study previously reported cost-effectiveness calculation using
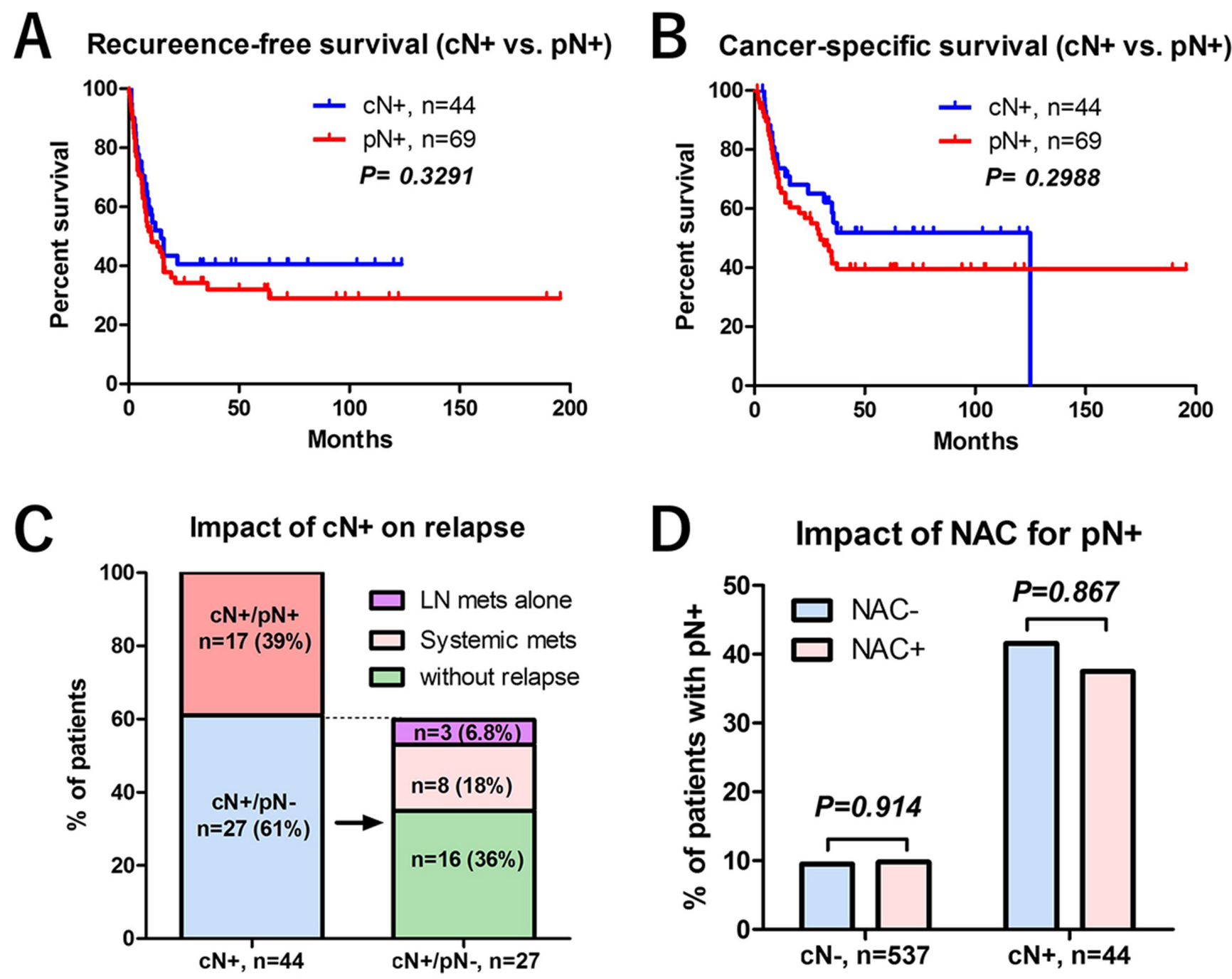

D Impact of NAC for $\mathrm{pN}+$



Figure 4: The impact of lymph node positive $(\mathrm{cN}+$ or $\mathbf{p N}+)$ on prognosis. (A) Recurrence-free survival between the patients with $\mathrm{cN}+$ and $\mathrm{pN}+$ was not significantly different. (B) Cancer-specific survival between the patients with $\mathrm{cN}+$ and $\mathrm{pN}+$ was not significantly different. (C) The impact of $\mathrm{cN}+$ on tumor recurrence was shown. Of 44 patients with $\mathrm{cN}+$, only $17(39 \%)$ patients had $\mathrm{cN}+$ and $\mathrm{pN}+$. Of 27 (61\%) patients with $\mathrm{cN}+/ \mathrm{pN}-, 11$ patients (25\%) experienced recurrence. Three patients with $\mathrm{cN}+/ \mathrm{pN}-(6.8 \%)$ had lymph nodes recurrence alone while remain $8(18 \%)$ had systemic recurrence. (D) There were no significant difference in the frequencies of pN+between $\mathrm{cN}$ patients treated with NAC $(31 / 316,9.8 \%)$ and without NAC $(21 / 221,9.5 \%)(P=0.914)$, and $\mathrm{pN}+$ between $\mathrm{cN}+$ treated with NAC $(12 / 32$, $38 \%)$ and without NAC $(5 / 12,42 \%)(P=0.867)$. 
risk-score based protocol. We believe our study provides the idea for risk stratification to improve the cost-effectiveness. A prospective study on the cost effectiveness of followup using a universal, standard, and easily applicable surveillance model is required to validate these results.

In conclusion, a risk-score-stratified surveillance protocol has the potential to reduce over investigation during follow-up making surveillance more cost effective. Further study is needed to determine the impact of riskstratification on the cost effectiveness of oncological follow-up after RC.

\section{MATERIALS AND METHODS}

\section{Ethics statement}

This retrospective study was performed following the ethical standards of the Declaration of Helsinki, and was approved by the Ethics Committee of the Hirosaki University School of Medicine (authorization numbers 2015-258 and 2016-225).

\section{Patient selection}

Between May 1996 and December 2016, a consecutive series of 581 adults underwent RC and urinary diversion at Hirosaki university hospital, Aomori Rosai Hospital, Mutsu General Hospital, and Aomori Prefectural Central Hospital.

\section{Patient variables}

The variables analyzed were age, sex, Eastern Cooperative Oncology Group performance status (ECOG PS), clinical stage, renal function, and history of hypertension (HTN), cardiovascular disease (CVD), and diabetes mellitus (DM). CVD was defined as a positive history of cardiac surgery, angina, myocardial infarction, stroke, or taking any cardiotonic agents and/or coronary vasodilators. Diabetic patients were defined as those with a history of type 2 diabetes or those who met the relevant diagnostic criteria and required glycemic control. Renal function was evaluated by estimated glomerular filtration rate (eGFR) using a modified version of the abbreviated Modification of Diet in Renal Disease Study formula for Japanese patients [41]. Chronic kidney disease (CKD) criteria included a preoperative eGFR $<60 \mathrm{~mL} / \mathrm{min} / 1.73$ $\mathrm{m}^{2}$. Tumor stage and grade were defined by the 2009 TNM classification of the Union of International Cancer Control [42]. We used computed tomography (CT) and cystoscopy for preoperative TNM classification. Magnetic resonance imaging (MRI) and bone scintigraphy were indicated for
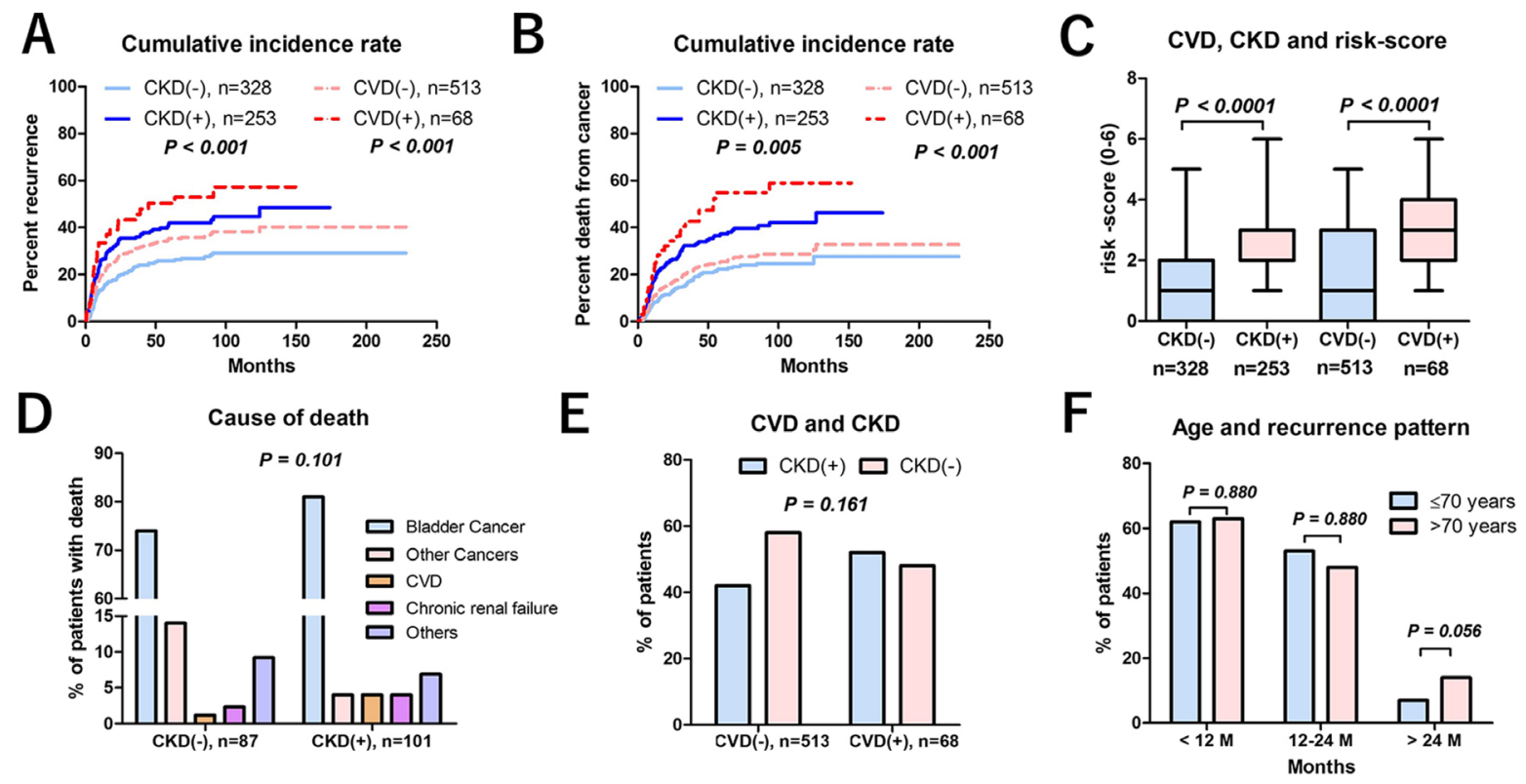

Figure 5: The impact of chronic kidney disease (CKD), cardiovascular disease (CVD), or age on prognosis. (A) Recurrence-free survival of the patients with CKD was significantly shorter than the patients without CKD. Recurrence-free survival in the patients with CVD was significantly shorter than the patients without CVD. (B) Cancer-specific survival of the patients with CKD was significantly shorter than the patients without CKD. Cancer-specific survival in the patients with CVD was significantly shorter than the patients without CVD. (C) The patients with CKD had significantly higher risk-score $(2.5 \pm 1.3$ points) than those without CKD (1.2 \pm 1.3 points, $P<0.001)$ (Figure $5 \mathrm{C}$, left). Similarly, the patients with CVD had significantly higher risk-score $(3.1 \pm 1.3$ points) than those without CVD (1.6 \pm 1.4 points, $P<0.001)$ (Figure 5C, right). (D) No difference was observed in the cause of death between patients with and without CKD. (E) There was no significant difference between the number of CKD patients with CVD $(n=35 / 68,52 \%)$ and without CVD $(n=218 / 513,42 \%)$. (F) The relationship between age and recurrence pattern showed no significantly difference between the patients with $\leq 70$ and with $>70$ years old. 
the selected patients with when advanced disease $(\geq \mathrm{T} 3 \mathrm{~b}$ and/or $\mathrm{cN}+$ ) was suspected. Postoperative complications were evaluated by the Clavien-Dindo classification [43].

\section{Neoadjuvant chemotherapy (NAC)}

We routinely used 2 or 3 NAC regimens for MIBC patients after 2005. The exclusion criteria for NAC in our institute include patients with chronic active hepatitis, severe liver dysfunction, severe chronic obstructive pulmonary disease with home oxygen therapy, severe chronic heart failure, or undergoing dialysis. These were a platinum-based combination regimens using either gemcitabine plus cisplatin (GCis), gemcitabine plus carboplatin $(\mathrm{GCb})$, or methotrexate, vinblastine, adriamycin, and cisplatin (MVAC). Regimens were selected based on guidelines regarding eligibility for the proper use of cisplatin [44] and a patient's overall status.

\section{Surgical procedure}

All patients experienced RC, urinary diversion, and a standard pelvic lymph node dissection (PLND) that included the removal of the obturator, external iliac, hypogastric, and common iliac lymph node chains (there were no para-aortic or paracaval dissections). All RCs were performed by high-volume surgeons $(>15$ cases per year, or $>100$ cases in recent 10 years) using the same basic technique [45]. Orthotopic ileal neobladder construction, ileal conduit diversion, and cutaneous ureterostomy were performed by previously reported methods [46-48].

\section{Follow-up surveillance protocol}

Oncological follow-up after RC was performed following National Comprehensive Cancer Network and European Association of Urology guidelines and previously published pathology protocols $[4,9]$. Based on pathological outcome after RC, all patients were stratified for oncological follow-up to normal-risk ( $\leq$ pT2N0) and high-risk ( $\geq$ pT3N0, pN+, lymphovascular invasion positive (LVI+), or histological variants) groups. The pathology-based protocol is shown in Table 1 (upper rows). Follow-up of high-risk patients was recommended every 3 months for the first 2 years after surgery, semiannually for the next 3 years, and annually thereafter, barring evidence of disease recurrence. Follow-up of normal-risk patients was recommended every 3 months for the first year and every 6 months for the following 3 years and annually thereafter in patients without any evidence of disease recurrence. Follow-up evaluation included urine cytology and ultrasonography every 3-6 months to monitor urothelial recurrence and hydronephrosis in both groups. Blood biochemistry, and CT of the chest/ abdomen/pelvis were performed every 3-6 months for at least 5 years to monitor serum electrolytes, blood urea nitrogen, serum creatinine, and liver function. Evaluations by bone scans or brain imaging were performed when clinically indicated. Disease recurrence was classified as in the lymph nodes, visceral organs, local pelvis, bones, urothelium (urethra plus upper urinary tract), or brain. Lymph node recurrence included metastasis to local pelvic, paraaortic, thoracic, mediastinal, and paratracheal lymph nodes. Visceral organ recurrence included metastasis to the liver, lungs, adrenal glands, and other intra-abdominal organs. The first recurrence after RC was recorded.

\section{Adjuvant and salvage therapy after radical cystectomy}

Adjuvant chemotherapy was not routinely administered. Systemic chemotherapy after recurrence consisted of a platinum-based combination regimen with GCis, GCb, gemcitabine, carboplatin and docetaxel (GCD), docetaxel, ifosfamide and nedaplatin (DIN), or MVAC. Regimens were selected based on residual renal function and overall status. Vital status was identified from death certificates or physician correspondence. For patients followed elsewhere, the cystectomy registry at our institution collected annual outcomes from the patient and treating physician.

\section{Outcome measurements}

Recurrence-free survival, time to recurrence, and estimated cost per one recurrence detection by the pathology-based protocol were recorded for the normaland high-risk groups. To estimate cost-benefit, we calculated the medical cost of follow-up to detect one recurrence $(=$ [screening cost in a follow-up period] / [number of patients with recurrence]) using an exchange rate of 100 yen to the U.S. dollar. Estimated medical costs were $\$ 350$ for a CT, $\$ 70$ for blood testing, $\$ 53$ for ultrasonography, and $\$ 25$ for urine cytology. The cost of prescriptions, medications, and doctor fees were not included in the analysis. To compare cost effectiveness, we developed a novel risk score-based protocol using a multivariate Cox proportional hazard regression model. Patient risk-scores were calculated by summing the number of independent risks suggested in the multivariate analysis, and the patients were stratified into low (0-1), intermediate (2-3), and high (4-6) (Table 4). Then, we developed our ad-hoc surveillance protocol using real incidences of recurrence after radical cystectomy (Table 1, lower columns). When the incidence of recurrence was frequent at a certain period, we defined the routine screening is necessary in this period. On the other hand, when the incidence of recurrence was not frequent ( 0 or 1$)$ at a certain period, we defined the routine screening is not necessary in this period. Based on this rule, we developed our surveillance protocol that enhancing the costeffectiveness without increasing the number of patients 
who were not detected by screening. The estimated cost per one recurrence detection in the pathology-based and risk score-based protocols were compared.

\section{Statistical analysis}

Statistical analysis was performed using SPSS version. 24.0 (SPSS, IBM Japan, Tokyo, JPN) and GraphPad Prism 5.03 (GraphPad Software, San Diego, CA, USA). Categorical variables were compared using Fisher's exact test or the chi-square test. Differences between groups were compared using $t$-tests for normally distributed values or the Mann-Whitney $U$-test for values that were not normally distributed. The Kruskal-Wallis test was used to compare medians among three groups. All tests were two-sided, and a $P$ value $<0.05$ was considered statistically significant. Overall survival in patients with recurrence, stratified by risk criteria was estimated using the Kaplan-Meier method and compared by the log rank test. Cox proportional hazard regression models were used to identify factors independently associated with recurrence-free survival; hazard ratios (HRs) with 95\% confidence intervals (CIs) were calculated after controlling simultaneously for potential confounders, including patient demographic and clinicopathological variables. Model variables included age, sex, ECOG PS, history of HTN, DM, CVD, preoperative CKD, NAC, urinary diversion, $\geq \mathrm{pT} 3$ or $\mathrm{SM}+, \mathrm{pN}+, \mathrm{LVI}+$, and nonurothelial carcinoma components.

\section{Ethical standards}

This study was performed in accordance with the ethical standards of the Declaration of Helsinki and approved by an ethics review board of Hirosaki University School of Medicine (authorization numbers; 2016-225 and 2015-258).

\section{Authors' contributions}

Conception and design: Shingo Hatakeyama; Acquisition of data: Ayumu Kusaka, Itsuto Hamano, Shingo Hatakeyama, Shogo Hosogoe, Hiromichi Iwamura, Naoki Fujita, Ken Fukushi, Takuma Narita, Hayato Yamamoto, Hiroyuki Ito, Kazuaki Yoshikawa, Toshiaki Kawaguchi, Kazuhisa Hagiwara; Analysis and interpretation of data: Ayumu Kusaka, Itsuto Hamano, Shingo Hatakeyama, Takahiro Yoneyama, Yasuhiro Hashimoto; Drafting of the manuscript: Ayumu Kusaka, Itsuto Hamano, Shingo Hatakeyama; Critical revision of the manuscript: Chikara Ohyama, Takuya Koie; Statistical analysis: Shingo Hatakeyama, Tohru Yoneyama; Obtaining funding: Shingo Hatakeyama, Chikara Ohyama; Administrative, technical, or material support: Yuki Tobisawa, Tohru Yoneyama.

\section{ACKNOWLEDGMENTS}

We thank Yuki Fujita, Yukie Nishizawa, Kaname Higuchi, Satomi Sakamoto, and Masako Isono for their invaluable help with data and sample collection. The authors would like to thank Enago (www.enago.jp) for the English language review.

\section{CONFLICTS OF INTEREST}

The authors declare no conflicts of interests.

\section{FINANCIAL DISCLOSURE}

This work was supported by a Grant-in-Aid for Scientific Research (No. 23791737, 24659708, 22390301, 15H02563, 15K15579, and 17K11119) from the Japan Society for the Promotion of Science.

\section{REFERENCES}

1. Studer UE, Burkhard FC, Schumacher M, Kessler TM, Thoeny H, Fleischmann A, Thalmann GN. Twenty years experience with an ileal orthotopic low pressure bladder substitute-lessons to be learned. J Urol. 2006; 176:161-6. https://doi.org/ 10.1016/s0022-5347(06)00573-8.

2. Hautmann RE, Abol-Enein H, Hafez K, Haro I, Mansson W, Mills RD, Montie JD, Sagalowsky AI, Stein JP, Stenzl A, Studer UE, Volkmer BG. Urinary diversion. Urology. 2007; 69:17-49. https://doi.org/ 10.1016/j.urology.2006.05.058.

3. Stein JP, Lieskovsky G, Cote R, Groshen S, Feng AC, Boyd S, Skinner E, Bochner B, Thangathurai D, Mikhail M, Raghavan D, Skinner DG. Radical cystectomy in the treatment of invasive bladder cancer: long-term results in 1,054 patients. J Clin Oncol. 2001; 19:666-75. https://doi. org/ 10.1200/JCO.2001.19.3.666.

4. Yafi FA, Aprikian AG, Fradet Y, Chin JL, Izawa J, Rendon R, Estey E, Fairey A, Cagiannos I, Lacombe L, Lattouf JB, Bell D, Saad F, et al. Surveillance guidelines based on recurrence patterns after radical cystectomy for bladder cancer: the Canadian Bladder Cancer Network experience. BJU Int. 2012; 110:1317-23. https://doi.org/ 10.1111/j.1464-410X.2012.11133.x.

5. Stenzl A, Cowan NC, De Santis M, Kuczyk MA, Merseburger AS, Ribal MJ, Sherif A, Witjes JA, European Association of Urology (EAU). Treatment of muscleinvasive and metastatic bladder cancer: update of the EAU guidelines. Eur Urol. 2011; 59:1009-18. https://doi.org/ 10.1016/j.eururo.2011.03.023.

6. Volkmer BG, Schnoeller T, Kuefer R, Gust K, Finter F, Hautmann RE. Upper urinary tract recurrence after radical cystectomy for bladder cancer--who is at risk? J Urol. 2009; 182:2632-7. https://doi.org/ 10.1016/j.juro.2009.08.046. 
7. Giannarini G, Kessler TM, Thoeny HC, Nguyen DP, Meissner C, Studer UE. Do patients benefit from routine follow-up to detect recurrences after radical cystectomy and ileal orthotopic bladder substitution? Eur Urol. 2010; 58:486-94. https://doi.org/ 10.1016/j.eururo.2010.05.041.

8. Vemana G, Vetter J, Chen L, Sandhu G, Strope SA. Sources of variation in follow-up expenditure after radical cystectomy. Urol Oncol. 2015; 33:267 e31-7. https://doi. org/ 10.1016/j.urolonc.2015.03.009.

9. Stewart-Merrill SB, Boorjian SA, Thompson RH, Psutka SP, Cheville JC, Thapa P, Bergstrahl EJ, Tollefson MK, Frank I. Evaluation of current surveillance guidelines following radical cystectomy and proposal of a novel risk-based approach. Urol Oncol. 2015; 33:339 e1-8. https://doi.org/ 10.1016/j.urolonc.2015.04.017.

10. Slaton JW, Swanson DA, Grossman HB, Dinney CP. A stage specific approach to tumor surveillance after radical cystectomy for transitional cell carcinoma of the bladder. J Urol. 1999; 162:710-4.

11. Kuroda M, Meguro N, Maeda O, Saiki S, Kinouchi T, Usami M, Kotake T. Stage specific follow-up strategy after cystectomy for carcinoma of the bladder. Int J Urol. 2002; 9:129-33.

12. Hamano I, Hatakeyama S, Iwamura H, Fujita N, Fukushi K, Narita T, Hagiwara K, Kusaka A, Hosogoe S, Yamamoto H, Tobisawa Y, Yoneyama T, Yoneyama T, et al. Preoperative chronic kidney disease predicts poor oncological outcomes after radical cystectomy in patients with muscle-invasive bladder cancer. Oncotarget. 2017; 8:61404-61414. https:// doi.org/10.18632/oncotarget.18248.

13. Yu HS, Hwang JE, Chung HS, Cho YH, Kim MS, Hwang EC, Oh KJ, Kim SO, Jung SI, Kang TW, Kwon DD, Park K, Ryu SB, et al. Is preoperative chronic kidney disease status associated with oncologic outcomes in upper urinary tract urothelial carcinoma? a multicenter propensity score-matched analysis. Oncotarget. 2017; 8:66540-66549. https://doi.org/10.18632/oncotarget.16239.

14. Nagai K, Sairenchi T, Irie F, Watanabe H, Ota H, Yamagata K. Relationship between Estimated Glomerular Filtration Rate and Cardiovascular Mortality in a Japanese Cohort with Long-Term Follow-Up. PLoS One. 2016; 11:e0156792. https://doi.org/ 10.1371/journal.pone.0156792.

15. Na SY, Sung JY, Chang JH, Kim S, Lee HH, Park YH, Chung W, Oh KH, Jung JY. Chronic kidney disease in cancer patients: an independent predictor of cancer-specific mortality. Am J Nephrol. 2011; 33:121-30. https://doi.org/ $10.1159 / 000323740$.

16. Yang Y, Li HY, Zhou Q, Peng ZW, An X, Li W, Xiong LP, Yu XQ, Jiang WQ, Mao HP. Renal Function and AllCause Mortality Risk Among Cancer Patients. Medicine (Baltimore). 2016; 95:e3728. https://doi.org/ 10.1097/ md.0000000000003728.

17. Wong G, Hayen A, Chapman JR, Webster AC, Wang JJ, Mitchell P, Craig JC. Association of CKD and cancer risk in older people. J Am Soc Nephrol. 2009; 20:1341-50. https:// doi.org/ 10.1681/asn.2008090998.

18. Cao J, Zhao X, Zhong Z, Zhang L, Zhu X, Xu R. Prognostic Value of Pre-operative Renal Insufficiency in Urothelial Carcinoma: A Systematic Review and Meta-Analysis. Sci Rep. 2016; 6:35214. https://doi.org/ 10.1038/srep35214.

19. Sha N, Xu H, Chen T, Tian DW, Xie WQ, Xie LG, Zhang Y, Xing C, Liu XT, Shen ZH, Wu ZL, Hu HL, Wu CL. The evaluation of the association between the metabolic syndrome and tumor grade and stage of bladder cancer in a Chinese population. Onco Targets Ther. 2016; 9:1175-9. https://doi.org/ 10.2147/ott.s102424.

20. Keller CR, Odden MC, Fried LF, Newman AB, Angleman S, Green CA, Cummings SR, Harris TB, Shlipak MG. Kidney function and markers of inflammation in elderly persons without chronic kidney disease: the health, aging, and body composition study. Kidney Int. 2007; 71:239-44. https:// doi.org/ 10.1038/sj.ki.5002042.

21. Rasool M, Ashraf MA, Malik A, Waquar S, Khan SA, Qazi MH, Ahmad W, Asif M, Khan SU, Zaheer A, Qaisrani MM, Khan AR, Iqbal A, et al. Comparative study of extrapolative factors linked with oxidative injury and anti-inflammatory status in chronic kidney disease patients experiencing cardiovascular distress. PLoS One. 2017; 12:e0171561. https://doi.org/ 10.1371/journal. pone. 0171561 .

22. Chowdhury R, Peel NM, Krosch M, Hubbard RE. Frailty and chronic kidney disease: A systematic review. Arch Gerontol Geriatr. 2017; 68:135-42. https://doi.org/ 10.1016/j.archger.2016.10.007.

23. Ballew SH, Chen Y, Daya NR, Godino JG, Windham BG, McAdams-DeMarco M, Coresh J, Selvin E, Grams ME. Frailty, Kidney Function, and Polypharmacy: The Atherosclerosis Risk in Communities (ARIC) Study. Am J Kidney Dis. 2017; 69:228-36. https://doi.org/ 10.1053/j. ajkd.2016.08.034.

24. Forman DE, Alexander KP. Frailty: A Vital Sign for Older Adults With Cardiovascular Disease. Can J Cardiol. 2016; 32:1082-7. https://doi.org/ 10.1016/j.cjca.2016.05.015.

25. Mandelblatt JS, Cai L, Luta G, Kimmick G, Clapp J, Isaacs C, Pitcher B, Barry W, Winer E, Sugarman S, Hudis C, Muss H, Cohen HJ, et al. Frailty and long-term mortality of older breast cancer patients: CALGB 369901 (Alliance). Breast Cancer Res Treat. 2017; 164:107-17. https://doi.org/10.1007/s10549-017-4222-8.

26. Pamoukdjian F, Aparicio T, Zelek L, Boubaya M, Caillet P, Francois V, de Decker L, Levy V, Sebbane G, Paillaud E. Impaired mobility, depressed mood, cognitive impairment and polypharmacy are independently associated with disability in older cancer outpatients: The prospective Physical Frailty in Elderly Cancer patients (PF-EC) cohort study. J Geriatr Oncol. 2017; 8:190-95. https://doi.org/10.1016/j. jgo.2017.02.003.

27. Nishijima TF, Deal AM, Williams GR, Guerard EJ, Nyrop KA, Muss HB. Frailty and inflammatory markers in 
older adults with cancer. Aging (Albany NY). 2017; 9:650 64. https://doi.org/ 10.18632/aging.101162.

28. Sato $T$, Hatakeyama S, Okamoto $T$, Yamamoto $H$, Hosogoe S, Tobisawa Y, Yoneyama T, Hashiba E, Yoneyama T, Hashimoto Y, Koie T, Hirota K, Ohyama C. Slow Gait Speed and Rapid Renal Function Decline Are Risk Factors for Postoperative Delirium after Urological Surgery. PLoS One. 2016; 11:e0153961. https://oi.org/ 10.1371/journal.pone.0153961.

29. Dogliotti L, Carteni G, Siena S, Bertetto O, Martoni A, Bono A, Amadori D, Onat H, Marini L. Gemcitabine plus cisplatin versus gemcitabine plus carboplatin as first-line chemotherapy in advanced transitional cell carcinoma of the urothelium: results of a randomized phase 2 trial. Eur Urol. 2007; 52:134-41. https://doi.org/ 10.1016/j. eururo.2006.12.029.

30. Koie T, Ohyama C, Hashimoto Y, Hatakeyama S, Yamamoto H, Yoneyama T, Kamimura N. Efficacies and safety of neoadjuvant gemcitabine plus carboplatin followed by immediate cystectomy in patients with muscleinvasive bladder cancer, including those unfit for cisplatin: a prospective single-arm study. Int J Clin Oncol. 2013; 18:724-30. https://doi.org/ 10.1007/s10147-012-0447-z.

31. Park JH, Lee SW, Kim HS, Kang SG, Ko YH, Kim ST, Kang SH, Park YJ, Choi IK, Oh SC, Sung DJ, Seo JH, Cheon J, et al. Combination of gemcitabine and carboplatin as first line treatment in elderly patients or those unfit for cisplatin-based chemotherapy with advanced transitional cell carcinoma of the urinary tract. Cancer Chemother Pharmacol. 2013; 71:1033-9. https://doi.org/ 10.1007/ s00280-013-2098-9.

32. Koie T, Ohyama C, Yamamoto H, Imai A, Hatakeyama S, Yoneyama T, Hashimoto Y, Yoneyama T, Tobisawa Y. Neoadjuvant gemcitabine and carboplatin followed by immediate cystectomy may be associated with a survival benefit in patients with clinical T2 bladder cancer. Med Oncol. 2014; 31:949. https://doi.org/ 10.1007/s12032-0140949-9.

33. Ohyama C, Hatakeyama S, Yoneyama $\mathrm{T}$, Koie $\mathrm{T}$. Neoadjuvant chemotherapy with gemcitabine plus carboplatin followed by immediate radical cystectomy for muscle-invasive bladder cancer. Int J Urol. 2014; 21:3-4. https://doi.org/ 10.1111/iju.12230.

34. Lerner SP. Bladder cancer: ASCO endorses EAU muscleinvasive bladder cancer guidelines. Nat Rev Urol. 2016; 13:440-1. https://doi.org/ 10.1038/nrurol.2016.114.

35. Alfred Witjes J, Lebret T, Comperat EM, Cowan NC, De Santis M, Bruins HM, Hernandez V, Espinos EL, Dunn J, Rouanne M, Neuzillet Y, Veskimae E, van der Heijden AG, et al. Updated 2016 EAU Guidelines on Muscle-invasive and Metastatic Bladder Cancer. Eur Urol. 2017; 71:462-75. https://doi.org/ 10.1016/j.eururo.2016.06.020.

36. Nicholson S. Chemotherapy for bladder cancer in patients with impaired renal function. Nat Rev Urol. 2011; 9:52-7. https://doi.org/ 10.1038/nrurol.2011.176.
37. Fahmy NM, Mahmud S, Aprikian AG. Delay in the surgical treatment of bladder cancer and survival: systematic review of the literature. Eur Urol. 2006; 50:1176-82. https://doi. org/ 10.1016/j.eururo.2006.05.046.

38. Fukushi K, Narita T, Hatakeyama S, Yamamoto $H$, Soma O, Matsumoto T, Tobisawa Y, Yoneyama T, Imai A, Yoneyama T, Hashimoto Y, Koie T, Ohyama C. Qualityof-life evaluation during platinum-based neoadjuvant chemotherapies for urothelial carcinoma. Int J Clin Oncol. 2017; 22:366-72. https://doi.org/ 10.1007/s10147-0161071-0.

39. Choi W, Porten S, Kim S, Willis D, Plimack ER, HoffmanCensits J, Roth B, Cheng T, Tran M, Lee IL, Melquist J, Bondaruk J, Majewski T, et al. Identification of distinct basal and luminal subtypes of muscle-invasive bladder cancer with different sensitivities to frontline chemotherapy. Cancer Cell. 2014; 25:152-65. https://doi.org/ 10.1016/j. ccr.2014.01.009.

40. McConkey DJ, Choi W, Dinney CP. Genetic subtypes of invasive bladder cancer. Curr Opin Urol. 2015; 25:449-58. https://doi.org/ 10.1097/mou.0000000000000200.

41. Matsuo S, Imai E, Horio M, Yasuda Y, Tomita K, Nitta K, Yamagata K, Tomino Y, Yokoyama H, Hishida A. Revised equations for estimated GFR from serum creatinine in Japan. Am J Kidney Dis. 2009; 53:982-92. https://doi.org/ 10.1053/j.ajkd.2008.12.034.

42. Sobin LH, Gospodarowicz MK, Wittekind C. TNM classification of malignant tumours. Wiley-Blackwell, 7th ed. 2009.

43. Clavien PA, Barkun J, de Oliveira ML, Vauthey JN, Dindo D, Schulick RD, de Santibanes E, Pekolj J, Slankamenac K, Bassi C, Graf R, Vonlanthen R, Padbury $R$, et al. The Clavien-Dindo classification of surgical complications: five-year experience. Ann Surg. 2009; 250:187-96. https://doi.org/ 10.1097/ SLA.0b013e3181b13ca2.

44. Galsky MD, Hahn NM, Rosenberg J, Sonpavde G, Hutson T, Oh WK, Dreicer R, Vogelzang N, Sternberg C, Bajorin DF, Bellmunt J. A consensus definition of patients with metastatic urothelial carcinoma who are unfit for cisplatinbased chemotherapy. Lancet Oncol. 2011; 12:211-4. https:// dx.doi.org/10.1016/S1470-2045(10)70275-8.

45. Koie T, Ohyama C, Yamamoto H, Hatakeyama S, Kudoh S, Yoneyama T, Hashimoto Y, Kamimura N. Minimum incision endoscopic radical cystectomy in patients with malignant tumors of the urinary bladder: clinical and oncological outcomes at a single institution. Eur J Surg Oncol. 2012; 38:1101-5. https://doi.org/ 10.1016/j. ejso.2012.07.115.

46. Koie T, Hatakeyama S, Yoneyama T, Ishimura H, Yamato T, Ohyama C. Experience and functional outcome of modified ileal neobladder in 95 patients. Int J Urol. 2006; 13:1175-9. https://doi.org/ 10.1111/j.1442-2042.2006.01525.x. 
47. Toyoda Y. A new technique for catheterless cutaneous ureterostomy. J Urol. 1977; 117:276-8.
48. Bricker EM. Bladder substitution after pelvic evisceration. Surg Clin North Am. 1950; 30:1511-21. 